\title{
Effect of Sitagliptin on Cardiovascular Outcomes in Type 2 Diabetes
}

\author{
Jennifer B. Green, M.D., M. Angelyn Bethel, M.D., Paul W. Armstrong, M.D., \\ John B. Buse, M.D., Ph.D., Samuel S. Engel, M.D., Jyotsna Garg, M.S., \\ Robert Josse, M.B., B.S., Keith D. Kaufman, M.D., Joerg Koglin, M.D., \\ Scott Korn, M.D., John M. Lachin, Sc.D., Darren K. McGuire, M.D., M.H.Sc., \\ Michael J. Pencina, Ph.D., Eberhard Standl, M.D., Ph.D., Peter P. Stein, M.D., \\ Shailaja Suryawanshi, Ph.D., Frans Van de Werf, M.D., Ph.D., \\ Eric D. Peterson, M.D., M.P.H., and Rury R. Holman, M.B., Ch.B., \\ for the TECOS Study Group*
}

From the Duke Clinical Research Institute, Duke University School of Medicine, Durham (J.B.G., J.G., M.J.P., E.D.P.) and University of North Carolina School of Medicine, Chapel Hill (J.B.B.) - both in North Carolina; Diabetes Trials Unit, Oxford Centre for Diabetes, Endocrinology and Metabolism, University of Oxford, Oxford, United Kingdom (M.A.B., R.R.H.); Canadian VIGOUR Centre, University of Alberta, Edmonton, AB (P.W.A.) and St. Michael's Hospital, University of Toronto, Toronto (R.J.) — both in Canada; Merck, Kenilworth, NJ (S.S.E., K.D.K., J.K., S.K., P.P.S., S.S.); George Washington University Biostatistics Center, Rockville, MD (J.M.L.); University of Texas Southwestern Medical Center, Dallas (D.K.M.); Munich Diabetes Research Group, Helmholtz Center, Neuherberg, Germany (E.S.); and University of Leuven, Leuven, Belgium (F.V.W.). Address reprint requests to Dr. Holman at the Diabetes Trials Unit, Oxford Centre for Diabetes, Endocrinology and Metabolism, Churchill Hospital, Oxford OX3 7LJ, United Kingdom, or at rury.holman@dtu.ox.ac.uk.

*A complete list of members in the Trial Evaluating Cardiovascular Outcomes with Sitagliptin (TECOS) Study Group is provided in Supplementary Appendix 1, available at NEJM.org.

Drs. Green and Bethel contributed equally to this article.

This article was published on June 8, 2015, and updated on July 16, 2015, at NEJM.org.

N Engl J Med 2015;373:232-42.

DOI: $10.1056 /$ NEJMoal501352

Copyright (C) 2015 Massachusetts Medical Society.

\section{ABSTRACT}

\section{BACKGROUND}

Data are lacking on the long-term effect on cardiovascular events of adding sitagliptin, a dipeptidyl peptidase 4 inhibitor, to usual care in patients with type 2 diabetes and cardiovascular disease.

\section{METHODS}

In this randomized, double-blind study, we assigned 14,671 patients to add either sitagliptin or placebo to their existing therapy. Open-label use of antihyperglycemic therapy was encouraged as required, aimed at reaching individually appropriate glycemic targets in all patients. To determine whether sitagliptin was noninferior to placebo, we used a relative risk of 1.3 as the marginal upper boundary. The primary cardiovascular outcome was a composite of cardiovascular death, nonfatal myocardial infarction, nonfatal stroke, or hospitalization for unstable angina.

\section{RESULTS}

During a median follow-up of 3.0 years, there was a small difference in glycated hemoglobin levels (least-squares mean difference for sitagliptin vs. placebo, -0.29 percentage points; $95 \%$ confidence interval [CI], -0.32 to -0.27$)$. Overall, the primary outcome occurred in 839 patients in the sitagliptin group (11.4\%; 4.06 per 100 person-years) and 851 patients in the placebo group (11.6\%; 4.17 per 100 person-years). Sitagliptin was noninferior to placebo for the primary composite cardiovascular outcome (hazard ratio, $0.98 ; 95 \% \mathrm{CI}, 0.88$ to 1.09 ; $\mathrm{P}<0.001$ ). Rates of hospitalization for heart failure did not differ between the two groups (hazard ratio, $1.00 ; 95 \% \mathrm{CI}, 0.83$ to $1.20 ; \mathrm{P}=0.98$ ). There were no significant between-group differences in rates of acute pancreatitis $(\mathrm{P}=0.07)$ or pancreatic cancer $(\mathrm{P}=0.32)$.

\section{CONCLUSIONS}

Among patients with type 2 diabetes and established cardiovascular disease, adding sitagliptin to usual care did not appear to increase the risk of major adverse cardiovascular events, hospitalization for heart failure, or other adverse events. (Funded by Merck Sharp \& Dohme; TECOS ClinicalTrials.gov number, NCT00790205.) 
OOD GLYCEMIC CONTROL AMONG PAtients with type 2 diabetes reduces the risk of diabetes-related microvascular complications..$^{1-3}$ Many antihyperglycemic agents are licensed for the treatment of type 2 diabetes, but questions regarding the long-term cardiovascular safety of some of these agents have been raised. ${ }^{4,5}$ International regulatory agencies have responded by requiring that new antihyperglycemic agents not only show glucose-lowering ability but also are not associated with clinically meaningful increases in rates of major adverse cardiovascular events. ${ }^{6,7}$

Sitagliptin, an orally administered dipeptidyl peptidase 4 (DPP-4) inhibitor, prolongs the action of incretin hormones, including glucagonlike peptide 1 and glucose-dependent insulinotropic polypeptide, by inhibiting their breakdown. This improves glycemic control in patients with type 2 diabetes, primarily by suppressing glucagon levels and increasing endogenous insulin secretion. ${ }^{8}$ Two previous cardiovascular outcome trials of other DPP-4 inhibitors did not show an increase or decrease in the number of major adverse cardiovascular events but did raise safety concerns regarding a possible elevated risk of hospitalization for heart failure, ${ }^{9,10}$ with metaanalyses of randomized, controlled trials suggesting an increase of 24 to $25 \%$ in such a risk associated with these agents. ${ }^{11,12}$

In the Trial Evaluating Cardiovascular Outcomes with Sitagliptin (TECOS), we assessed the long-term cardiovascular safety of adding sitagliptin to usual care, as compared with usual care alone, in patients with type 2 diabetes and established cardiovascular disease.

\section{METHODS}

\section{STUDY OVERSIGHT}

We conducted this randomized, double-blind, placebo-controlled, event-driven trial at 673 sites in 38 countries on the basis of a design and rationale that have been reported previously. ${ }^{13}$ The study was designed and run independently by the Duke Clinical Research Institute (DCRI) and the University of Oxford Diabetes Trials Unit (DTU) in an academic collaboration with the sponsor, Merck Sharp \& Dohme. The trial organization, participating countries, and a list of investigators are provided in Supplementary Appendix 1 , available with the full text of this ar- ticle at NEJM.org. The protocol was approved by the ethics committee at each participating trial site. All analyses were performed by DCRI and DTU independent of the sponsor and according to the prespecified statistical analysis plan. The first and second authors wrote the first draft of the manuscript. Executive committee members and coauthors from DCRI, DTU, and the sponsor reviewed the data, revised the manuscript, and assume responsibility for trial adherence to the protocol and the accuracy and completeness of the data and the analyses. The protocol is also available at NEJM.org.

\section{STUDY POPULATION}

Eligible patients had type 2 diabetes with established cardiovascular disease and were at least 50 years of age, with a glycated hemoglobin level of 6.5 to $8.0 \%$ when treated with stable doses of one or two oral antihyperglycemic agents (metformin, pioglitazone, or sulfonylurea) or insulin (with or without metformin). Established cardiovascular disease was defined as a history of major coronary artery disease, ischemic cerebrovascular disease, or atherosclerotic peripheral arterial disease. Patients were excluded if they had taken a DPP-4 inhibitor, glucagon-like peptide-1 receptor agonist, or thiazolidinedione (other than pioglitazone) during the preceding 3 months; if they had a history of two or more episodes of severe hypoglycemia (defined as requiring thirdparty assistance) during the preceding 12 months; or if the estimated glomerular filtration rate (eGFR) was less than $30 \mathrm{ml}$ per minute per $1.73 \mathrm{~m}^{2}$ of body-surface area at baseline. All enrolled patients provided written informed consent.

\section{RANDOMIZATION AND STUDY MEDICATION}

Patients were randomly assigned in a 1:1 ratio to receive either sitagliptin at a dose of $100 \mathrm{mg}$ daily (or $50 \mathrm{mg}$ daily if the baseline eGFR was $\geq 30$ and $<50 \mathrm{ml}$ per minute per $1.73 \mathrm{~m}^{2}$ ) or matching placebo. An interactive voice-response system assigned the study medication in a double-blind manner, blocked within each site. This regimen was continued throughout the followup period, with predefined dose adjustments for changes in the eGFR. ${ }^{13}$ Patients who had two or more episodes of severe hypoglycemia between study visits, despite adjustment of other antihyperglycemic agents, were required to discontinue the study medication. 
The glycated hemoglobin level was measured locally at enrollment, at 4 and 8 months, and then annually. All other laboratory values of interest were collected opportunistically from usual-care data. Since sitagliptin lowers the glucose level, patients in the sitagliptin group would be expected to have lower glycated hemoglobin levels than those in the placebo group initially. During the study, the use of open-label antihyperglycemic agents was encouraged as required, with the aim of achieving individually appropriate glycated hemoglobin targets in all patients. This approach was taken to permit the assessment of possible drug-specific effects by minimizing potential confounding effects of differential glucose control. All the patients were followed until study closeout whenever possible, regardless of whether they were taking a study medication.

\section{OUTCOMES}

The primary composite cardiovascular outcome was defined as the first confirmed event of cardiovascular death, nonfatal myocardial infarction, nonfatal stroke, or hospitalization for unstable angina. The secondary composite cardiovascular outcome was the first confirmed event of cardiovascular death, nonfatal myocardial infarction, or nonfatal stroke.

Other secondary outcomes included the occurrence of the individual components of the primary composite cardiovascular outcome, fatal and nonfatal myocardial infarction, fatal and nonfatal stroke, death from any cause, and hospitalization for heart failure. Additional prespecified outcomes included changes in the glycated hemoglobin level and the eGFR, initiation of additional antihyperglycemic agents or long-term insulin therapy, and frequency of severe hypoglycemia. An independent clinicalevents classification committee whose members were unaware of study-group assignments adjudicated all events of death, myocardial infarction, stroke, hospitalization for unstable angina, hospitalization for heart failure, acute pancreatitis, and cancers (other than nonmelanoma skin cancers). All outcome definitions are provided in Supplementary Appendix 1. Adjudicated event definitions are listed in the Clinical Events Classification Committee Charter, which is available in Supplementary Appendix 2 at NEJM.org.

During the trial period, prespecified events of clinical interest that were collected systematically (including cardiovascular events), severe hypoglycemia, and expected diabetes-related complications were not reported as adverse events. Other serious adverse events were collected and reported according to regulatory requirements. Data regarding nonserious adverse events that were not part of prespecified outcomes were not collected.

\section{STATISTICAL ANALYSIS}

We assessed the primary noninferiority hypothesis by determining whether the upper boundary of the two-sided $95 \%$ confidence interval of the hazard ratio for the risk of the primary composite cardiovascular outcome did not exceed 1.30 in the sitagliptin group, as compared with the placebo group, in the per-protocol population, with a key supporting analysis in the intentionto-treat population. The statistical analysis plan prespecified that the following hypotheses be tested in a sequential manner: noninferiority for the primary composite cardiovascular outcome (main analysis, per-protocol; supporting analysis, intention-to-treat), noninferiority for the secondary composite cardiovascular outcome in the per-protocol analysis, superiority for the primary composite cardiovascular outcome in the intention-to-treat analysis, and superiority for the secondary composite cardiovascular outcome in the intention-to-treat analysis. We used the Cox proportional-hazards model to calculate hazard ratios and two-sided 95\% confidence intervals, stratified according to region. For this analysis, data from all patients were censored on the date they were last known to be free of the components of the primary composite cardiovascular outcome. We performed sensitivity analyses to assess the effect of missing data on study results on the basis of inverse weighting of the probability of dropout. ${ }^{14}$

We calculated that 611 patients with a confirmed primary composite cardiovascular outcome would provide a power of $90 \%$ for the test of noninferiority (hazard ratio, 1.00). For superiority, we calculated that 1300 patients with a primary composite cardiovascular outcome would provide a power of approximately $81 \%$ to determine the superiority of sitagliptin over placebo (hazard ratio, 0.85). Assuming an annual rate of 2.5 to $3.0 \%$ for the primary composite cardiovascular outcome, we determined that the 
randomization of approximately 14,000 patients with a 6-year study period in total would yield at least 1300 patients with the primary composite cardiovascular outcome.

We summarized the baseline characteristics of the patients using means $( \pm S D)$ or medians and interquartile ranges. Prespecified subgroups were analyzed on the basis of the previous type of oral antihyperglycemic agent received, previous insulin use, race, geographic region, sex, age at randomization, glycated hemoglobin level, duration of diabetes, body-mass index, renal function, smoking status, history of hypertension, blood pressure, previous heart failure, and use of antihypertensive agents, statins, or aspirin.

We performed additional analyses of the first hospitalization for heart failure and the composite of hospitalization for heart failure or cardiovascular death in both the per-protocol and intention-to-treat populations, with methods analogous to those used for the analysis of the primary composite cardiovascular outcome. Further details regarding the statistical analysis plan, including the definition of the per-protocol population, are provided in the study protocol. All analyses were conducted with the use of SAS software, version 9.0 or higher (SAS Institute).

\section{RESULTS}

\section{STUDY PATIENTS}

A total of 14,735 patients underwent randomization from December 2008 through July 2012. Of these patients, 14,671 were included in the intention-to-treat population, with 7332 assigned to receive sitagliptin and 7339 assigned to receive placebo (Fig. 1). The study was closed in March 2015, after the requisite minimum of 1300 patients were confirmed to have had a primary composite outcome. Median follow-up was 3.0 years (interquartile range, 2.3 to 3.8 ; maximum, 5.7). Overall, $95.1 \%$ of patients in the sitagliptin group and $94.1 \%$ of those in the placebo group completed the study, with $26.1 \%$ and $27.5 \%$ of all study patients, respectively, discontinuing study medication prematurely. Vital status was obtained for $97.5 \%$ of patients (Fig. 1). The characteristics of the patients at baseline were well balanced between the study groups with respect to demographic characteristics and the use of antihyperglycemic agents and secondary cardio- vascular prevention medications (Table S1 in Supplementary Appendix 1). At baseline, the mean $( \pm S D)$ glycated hemoglobin level was $7.2 \pm 0.5 \%$, and patients had been living with diabetes for a mean of $11.6 \pm 8.1$ years.

\section{GLYCEMIC CONTROL}

At 4 months, the mean glycated hemoglobin values were 0.4 percentage points lower in the sitagliptin group than in the placebo group. This difference narrowed during the study period, with an overall least-squares mean difference of $-0.29 \%$ in the sitagliptin group ( $95 \%$ confidence interval [CI], -0.32 to -0.27 ) (Fig. 2). Patients in the sitagliptin group received fewer additional antihyperglycemic agents than did those in the placebo group during the study period (1591 vs. 2046 patients; hazard ratio, 0.72; 95\% CI, 0.68 to $0.77 ; \mathrm{P}<0.001)$ and were less likely to start long-term insulin therapy (542 vs. 744 patients; hazard ratio, $0.70 ; 95 \% \mathrm{CI}, 0.63$ to $0.79 ; \mathrm{P}<0.001$ ) (Table S2 in Supplementary Appendix 1).

\section{PRIMARY AND SECONDARY COMPOSITE CARDIOVASCULAR OUTCOMES}

Overall in the intention-to-treat population, the primary composite cardiovascular outcome occurred in 839 patients in the sitagliptin group (11.4\%, 4.06 per 100 person-years) and 851 in the placebo group (11.6\%, 4.17 per 100 personyears). There was no significant between-group difference in the primary composite cardiovascular outcome (hazard ratio in the per-protocol analysis, 0.98 ; $95 \% \mathrm{CI}, 0.88$ to 1.09 ; $\mathrm{P}<0.001$ for noninferiority; hazard ratio in the intention-totreat analysis, $0.98 ; 95 \% \mathrm{CI}, 0.89$ to $1.08 ; \mathrm{P}=0.65$ for superiority) or in the secondary composite cardiovascular outcome (hazard ratio in the perprotocol analysis, 0.99 ; 95\% CI, 0.89 to 1.11 ; $\mathrm{P}<0.001$ for noninferiority; hazard ratio in the intention-to-treat analysis, $0.99 ; 95 \% \mathrm{CI}, 0.89$ to 1.10; $\mathrm{P}=0.84$ for superiority) (Table 1 and Fig. $3 \mathrm{~A}$ and $3 \mathrm{~B}$ ). Results from all sensitivity analyses were consistent with the findings in the main analyses. (All cardiovascular outcomes in the per-protocol analysis are provided in Table S3 in Supplementary Appendix 1.)

\section{OTHER SECONDARY OUTCOMES}

There was no significant difference in the rate of hospitalization for heart failure, which was reported in 228 patients in the sitagliptin group 


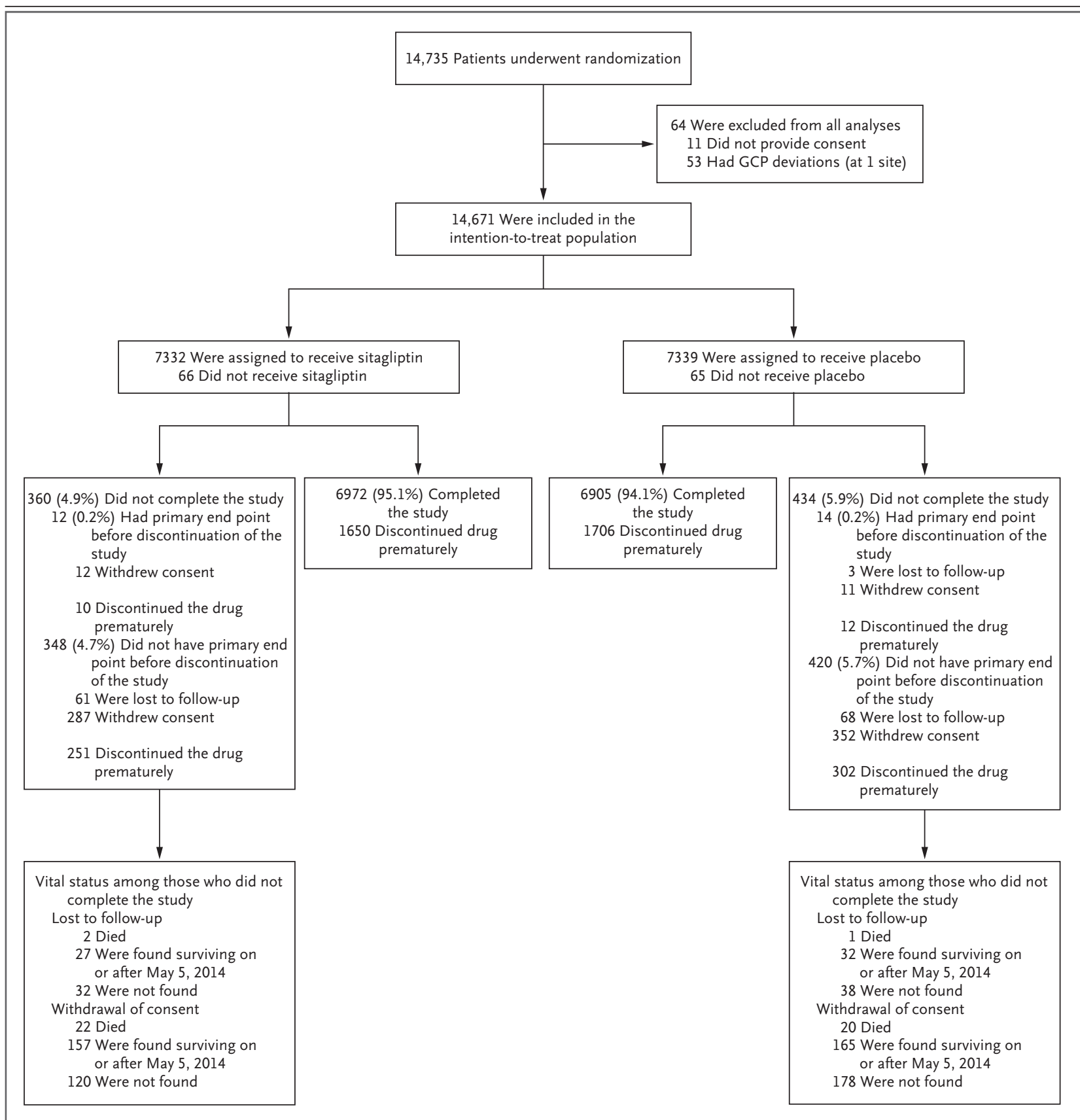

Figure 1. Enrollment, Follow-up, and Vital Status.

GCP denotes Good Clinical Practice guidelines.

(3.1\%; 1.07 per 100 person-years) and 229 in the placebo group (3.1\%; 1.09 per 100 person-years) (hazard ratio in the intention-to-treat analysis, 1.00 ; $95 \% \mathrm{CI}, 0.83$ to 1.20 ; $\mathrm{P}=0.98$ ). The composite outcome of hospitalization for heart failure or cardiovascular death occurred in $538 \mathrm{pa}-$ tients in the sitagliptin group $(7.3 \% ; 2.54$ per
100 person-years) and 525 in the placebo group (7.2\%; 2.50 per 100 person-years) (hazard ratio in the intention-to-treat analysis, 1.02; $95 \% \mathrm{CI}$, 0.90 to 1.15 ; $\mathrm{P}=0.74)$. Death from any cause occurred in 547 patients in the sitagliptin group (7.5\%; 2.48 per 100 person-years) and 537 in the placebo group (7.3\%; 2.45 per 100 person-years) 


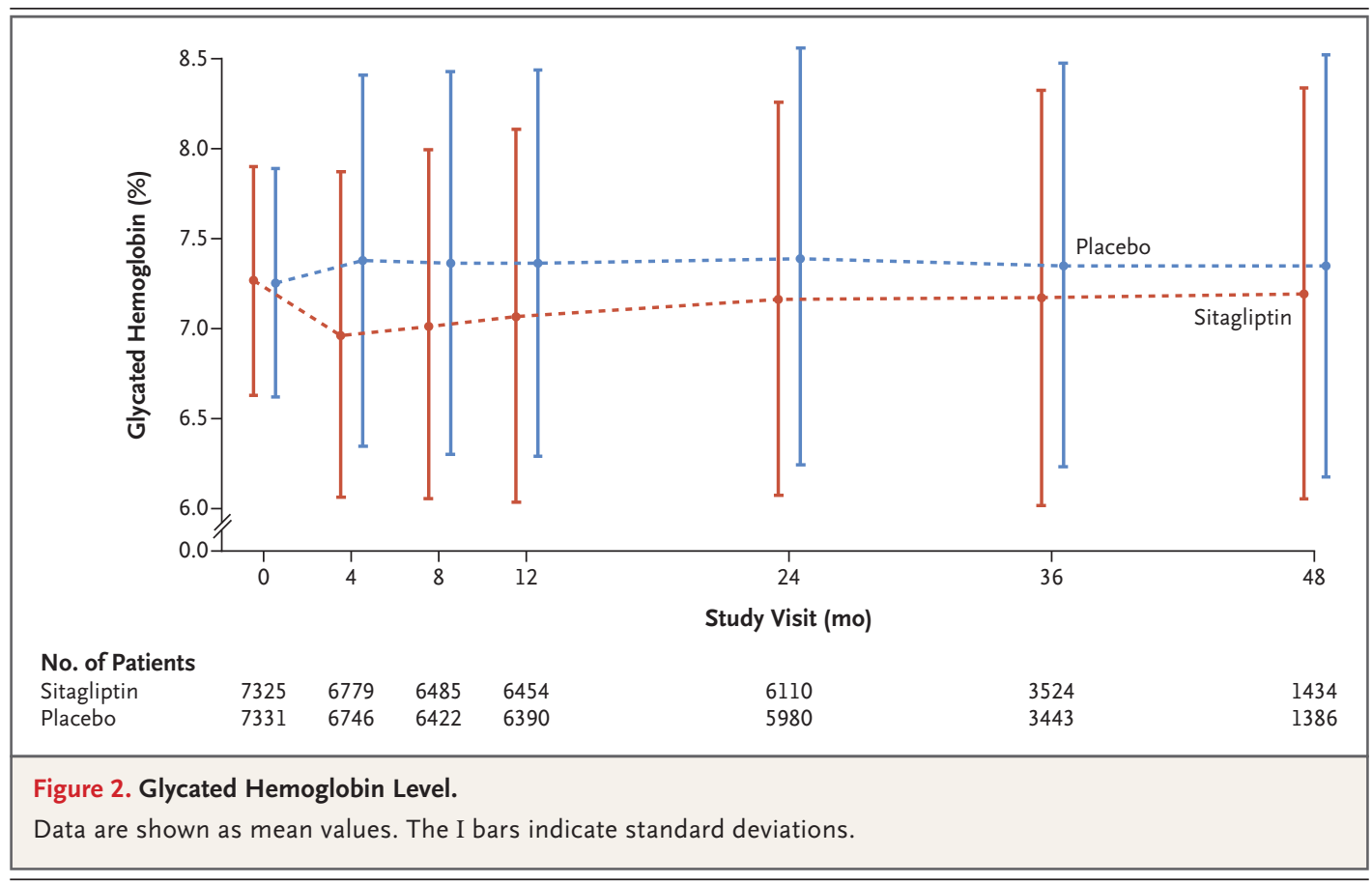

(hazard ratio in the intention-to-treat analysis, 1.01; 95\% CI, 0.90 to 1.14; $\mathrm{P}=0.88$ ) (Table 1 and Fig. 3C and 3D).

\section{SUBGROUP ANALYSES}

The results of subgroup analyses of major prespecified primary cardiovascular outcomes are provided in Figure S1 in Supplementary Appendix 1. No significant interactions were observed apart from body-mass index.

\section{SAFETY OUTCOMES}

There was no significant difference between the sitagliptin group and the placebo group with respect to the overall incidence of infections, cancer, site-reported renal failure, or severe hypoglycemia. In the two study groups, patients who had at least one severe hypoglycemic episode had a longer mean duration of diabetes and were more often taking insulin. Confirmed acute pancreatitis events were uncommon overall but numerically more frequent in the sitagliptin group (23 events [0.3\%]) than in the placebo group (12 events [0.2\%]) $(\mathrm{P}=0.07$ in the intention-to-treat analysis and $\mathrm{P}=0.12$ in the per-protocol analysis) (Table S4 in Supplementary Appendix 1). Confirmed pancreatic cancers were also uncommon overall but numerically less frequent in the sitagliptin group (9 events
[0.1\%]) than in the placebo group (14 events $[0.2 \%])(\mathrm{P}=0.32$ in the intention-to-treat analysis and $\mathrm{P}=0.85$ in the per-protocol analysis) (Table 1 ).

The rate of death from noncardiovascular causes was $2.3 \%$ in the two study groups, with no notable differences in individual causes. Rates of death from infection were $0.6 \%$ and $0.7 \%$ in the sitagliptin group and the placebo group, respectively. No clinically relevant differences in the incidence of additional clinical events of interest or serious adverse events were noted (Table 2, and Table S5 in Supplementary Appendix 1).

At 48 months, the mean change from baseline in the eGFR was greater in the sitagliptin group than in the placebo group $(-4.0 \pm 18.4$ and $-2.8 \pm 18.3 \mathrm{ml}$ per minute per $1.73 \mathrm{~m}^{2}$, respectively). The slightly lower eGFR in the sitagliptin group remained consistent over all post-randomization visits, with an estimated least-squares mean difference of $-1.34 \mathrm{ml}$ per minute per 1.73 $\mathrm{m}^{2}$ (95\% CI, -1.76 to $\left.-0.91 ; \mathrm{P}<0.001\right)$.

\section{DISCUSSION}

In our global clinical trial, which was performed in a usual-care setting among patients with type 2 diabetes and established cardiovascular dis- 
Table 1. Rates of Composite Cardiovascular Outcomes and Key Secondary Outcomes.

Outcome

Per-protocol analysis
No. of patients in analysis
Cardiovascular outcome
Cardiovascular death, nonfatal myo-
cardial infarction, nonfatal
stroke, or hospitalization for
unstable angina: primary com
posite outcome
Cardiovascular death, nonfatal myo-
cardial infarction, or nonfatal
stroke: secondary composite
outcome

Noncardiovascular outcome

Acute pancreatitis

Charter-defined cancer

Pancreatic cancer

Severe hypoglycemia

Intention-to-treat analysis

No. of patients in analysis

Cardiovascular outcome

Cardiovascular death, nonfatal myocardial infarction, nonfatal stroke, or hospitalization for unstable angina: primary composite outcome

Cardiovascular death

Nonfatal myocardial infarction

Nonfatal stroke

Hospitalization for unstable angina

Cardiovascular death, nonfatal myocardial infarction, or nonfatal stroke: secondary composite outcome

Cardiovascular death

Nonfatal myocardial infarction

Nonfatal stroke

Secondary outcome

Cardiovascular death

Hospitalization for unstable angina

Fatal or nonfatal myocardial infarction

Fatal or nonfatal stroke

Death from any cause

Hospitalization for heart failure $\dagger$
Sitagliptin

no. (\%)

no. per 100

7257

person-yr

no. (\%)

Placebo

Hazard Ratio

(95\% CI)

P Value

no. per 100

7266

$695(9.6)$

3.73

$695(9.6)$

3.82

$0.98(0.88-1.09)$

$<0.001 *$

609 (8.4)

3.24

$602(8.3)$

3.28

$0.99(0.89-1.11)$

$<0.001 *$

$\begin{array}{cccccc}20(0.3) & 0.10 & 11(0.2) & 0.06 & 1.80(0.86-3.76) & 0.12 \\ 248(3.4) & 1.30 & 260(3.6) & 1.40 & 0.93(0.78-1.10) & 0.38 \\ 9(0.1) & 0.05 & 10(0.1) & 0.05 & 0.91(0.37-2.25) & 0.85 \\ 144(2.0) & 0.77 & 125(1.7) & 0.68 & 1.13(0.89-1.44) & 0.31\end{array}$

7332

7339

$839(11.4)$

4.06

$851(11.6)$

4.17

$0.98(0.89-1.08)$

0.65

$311(4.2)$

$291(4.0)$

275 (3.8)

286 (3.9)

$145(2.0)$

157 (2.1)

$108(1.5)$

$117(1.6)$

745 (10.2)

3.58

746 (10.2)

3.62

$0.99(0.89-1.10)$

0.84

$313(4.3)$

$293(4.0)$

285 (3.9)

$294(4.0)$

$147(2.0)$

$159(2.2)$

$380(5.2)$

1.72

$366(5.0)$

1.67

$1.03(0.89-1.19)$

0.71

116 (1.6)

0.54

129 (1.8)

0.61

$0.90(0.70-1.16)$

0.42

$300(4.1)$

1.42

$316(4.3)$

1.51

$0.95(0.81-1.11)$

0.49

$178(2.4)$

0.83

$183(2.5)$

0.87

0.97 (0.79-1.19)

0.76

547 (7.5)

2.48

537 (7.3)

2.45

1.01 (0.90-1.14)

0.88

$228(3.1)$

$229(3.1)$

$1.00(0.83-1.20)$

0.98

The New England Journal of Medicine

Downloaded from nejm.org at UNIVERSITY OF PISA on September 7, 2016. For personal use only. No other uses without permission. 


\begin{tabular}{|c|c|c|c|c|c|c|}
\hline \multirow[t]{2}{*}{ Outcome } & \multicolumn{2}{|c|}{ Sitagliptin } & \multicolumn{2}{|c|}{ Placebo } & \multirow[t]{2}{*}{$\begin{array}{l}\text { Hazard Ratio } \\
(95 \% \mathrm{Cl})\end{array}$} & \multirow[t]{2}{*}{ P Value } \\
\hline & no. (\%) & $\begin{array}{l}\text { no. per } 100 \\
\text { person-pr }\end{array}$ & no. (\%) & $\begin{array}{l}\text { no. per } 100 \\
\text { person- } y r\end{array}$ & & \\
\hline $\begin{array}{l}\text { Hospitalization for heart failure or } \\
\text { cardiovascular death } \uparrow^{-}\end{array}$ & $538(7.3)$ & 2.54 & $525(7.2)$ & 2.50 & $1.02(0.90-1.15)$ & 0.74 \\
\hline \multicolumn{7}{|l|}{ Noncardiovascular outcome } \\
\hline Acute pancreatitis & $23(0.3)$ & 0.11 & $12(0.2)$ & 0.06 & $1.93(0.96-3.88)$ & 0.07 \\
\hline Charter-defined cancer & $268(3.7)$ & 1.25 & $290(4.0)$ & 1.37 & $0.91(0.77-1.08)$ & 0.27 \\
\hline Pancreatic cancer & $9(0.1)$ & 0.04 & $14(0.2)$ & 0.07 & $0.66(0.28-1.51)$ & 0.32 \\
\hline Severe hypoglycemia & $160(2.2)$ & 0.78 & $143(1.9)$ & 0.70 & $1.12(0.89-1.40)$ & 0.33 \\
\hline
\end{tabular}

* The $\mathrm{P}$ value is for the noninferiority of sitagliptin, as compared with placebo, which was calculated by determining whether the upper boundary of the two-sided $95 \%$ confidence interval of the hazard ratio exceeded 1.30. All other listed $\mathrm{P}$ values are based on the Wald statistic from a Cox model stratified according to region with a test of differences in hazard ratios.

$\dagger$ The analyses of hospitalization for heart failure were adjusted for a history of heart failure at baseline.

ease, we found that the addition of sitagliptin to usual care among patients with glycemic equipoise did not affect rates of major atherosclerotic cardiovascular events. Sitagliptin therapy did not change rates of death from any cause, cardiovascular death, or noncardiovascular death, and there were no notable differences between the groups with regard to specific causes of death, including infection.

Sitagliptin therapy was not associated with changes in rates of hospitalization for heart failure (hazard ratio, 1.00), as has been suggested in trials of other DPP-4 inhibitors.,10 There were also no between-group differences in the rate of the composite outcome of hospitalization for heart failure or cardiovascular death.

No significant increase in the rate of severe hypoglycemia was seen among patients in the sitagliptin group, as compared with the placebo group. Fewer patients in the sitagliptin group required the use of additional antihyperglycemic agents or initiated long-term insulin therapy during the study period.

Concern has been raised about a possible association between incretin-based therapies and adverse pancreatic effects. ${ }^{15}$ Although acute pancreatitis was uncommon, it occurred more often in the sitagliptin group, but the difference was not significant. Pancreatic cancer was also uncommon and occurred more often in the placebo group, but again the difference was not significant.

The observation that sitagliptin therapy was not associated with a change in long-term rates of cardiovascular events is consistent with the findings from shorter-term outcome trials of other DPP-4 inhibitors, including saxagliptin and alogliptin. The Saxagliptin Assessment of Vascular Outcomes Recorded in Patients with Diabetes Mellitus-Thrombolysis in Myocardial Infarction 53 (SAVOR-TIMI 53) trial and the Examination of Cardiovascular Outcomes with Alogliptin versus Standard of Care (EXAMINE) trial both showed that these agents did not increase or decrease the number of major adverse cardiovascular events. ${ }^{9,10}$ However, results from the SAVOR-TIMI 53 trial showed an unexpected excess rate of hospitalization for heart failure in the saxagliptin group (hazard ratio, 1.27; 95\% CI, 1.07 to 1.51$).{ }^{8}$ The EXAMINE trial showed a nonsignificant numerical imbalance in hospitalization for heart failure in the alogliptin group as compared with placebo (hazard ratio, 1.19; $95 \%$ CI, 0.90 to 1.58 ) but no imbalance in a post hoc analysis of the composite of hospitalization for heart failure or cardiovascular death. ${ }^{16}$ In contrast, in our trial we found that rates of hospitalization for heart failure did not differ between the two groups. The reasons for the lack of a heart-failure safety signal in our trial as compared with previous trials of DPP-4 agents may relate to differences in the patients who were enrolled, in the background care that was provided, or in the recording and definition of heart-failure events, or to intrinsic pharmacologic differences among DPP-4 inhibitors, or it may simply represent the play of chance in previous findings. 


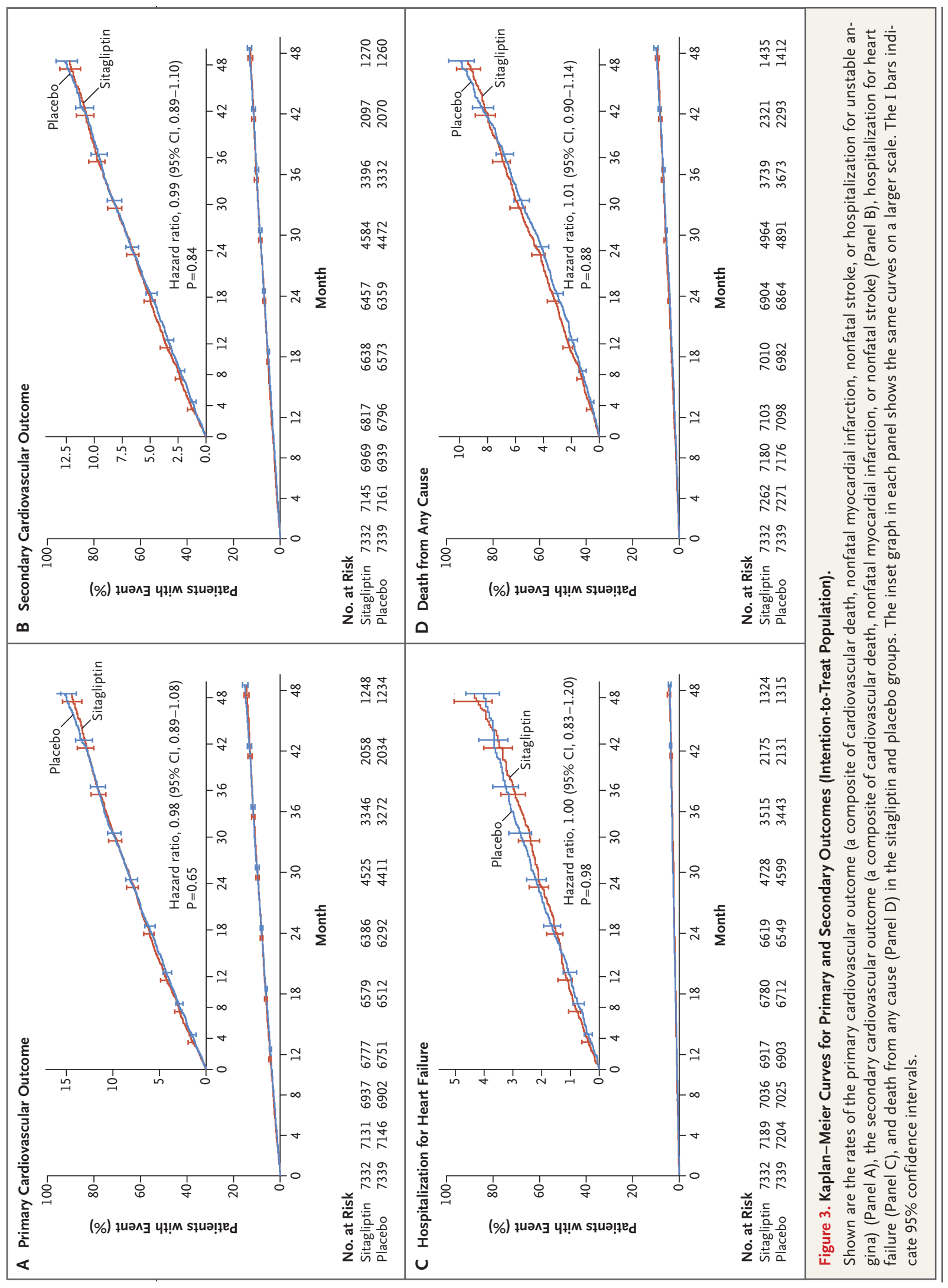




\begin{tabular}{|c|c|c|c|c|}
\hline \multirow[t]{2}{*}{ Event } & \multicolumn{2}{|c|}{$\begin{array}{l}\text { Sitagliptin } \\
(\mathrm{N}=7332)\end{array}$} & \multicolumn{2}{|c|}{$\begin{array}{l}\text { Placebo } \\
(\mathrm{N}=7339)\end{array}$} \\
\hline & no. of patients (\%) & no. of events & no. of patients (\%) & no. of events \\
\hline Benign, malignant, or unspecified neoplasm & $341(4.7)$ & 405 & $371(5.1)$ & 470 \\
\hline Injury, poisoning, or procedural complication & $146(2.0)$ & 165 & $133(1.8)$ & 153 \\
\hline Gastrointestinal disorder & $130(1.8)$ & 143 & $102(1.4)$ & 121 \\
\hline Musculoskeletal or connective-tissue disorder & $118(1.6)$ & 136 & $93(1.3)$ & 102 \\
\hline Respiratory, thoracic, or mediastinal disorder & $66(0.9)$ & 81 & $77(1.0)$ & 95 \\
\hline
\end{tabular}

* Adverse events that were reported in at least $1 \%$ of patients in either study group are listed according to the system organ class.

Our study was designed, run, and analyzed exclusively by the DCRI and the University of Oxford DTU, and only members of the data and safety monitoring board had access to unblinded outcome or efficacy data before the database lock. Our study was adequately powered, with greaterthan-anticipated event rates for the primary composite cardiovascular outcome, and had a longer follow-up than previous outcome studies of DPP-4 inhibitors. The population that we studied had well-managed cardiovascular and glycemic risk factors at baseline and was broadly similar to those studied in the SAVOR-TIMI 53 and EXAMINE trials. ${ }^{17}$ The results of our study would appear to have wide-ranging generalizability, given that it was carried out in a usual-care setting and included patients with a global distribution. There was uniform ascertainment and adjudication of hospitalization for heart failure, acute pancreatitis, and pancreatic cancer, with an aim of ensuring the integrity of the safety data.

Our study has certain limitations. We enrolled patients with moderate hyperglycemia (glycated hemoglobin level, 6.5 to $8.0 \%$ ) and excluded those with severe renal insufficiency. Potential biases are the possible confounding effects on cardiovascular outcomes by the small residual between-group difference in the glycated hemoglobin level and the greater use of antihyperglycemic agents in the placebo group. The opportunistic approach to data collection, apart from the selected values for glycated hemoglobin, resulted in limited acquisition of data regarding the ratio of urinary albumin to creatinine.

Our study results showed that sitagliptin may be used in a diverse group of patients with type 2 diabetes who are at high cardiovascular risk without increasing rates of cardiovascular complications, but these results cannot exclude possible benefits or risks with longer durations of therapy or in patients with more complicated coexisting illnesses. In our trial involving patients with type 2 diabetes and established cardiovascular disease, the addition of sitagliptin to usual care did not have a significant effect on rates of major adverse cardiovascular events or hospitalization for heart failure.

Supported by Merck Sharp \& Dohme, a subsidiary of Merck.

Disclosure forms provided by the authors are available with the full text of this article at NEJM.org.

We thank the patients, without whom this study and these analyses would not have been possible; and the following academic partners and contract research organizations for their assistance: Parexel International, Jubilant Clinsys, Clinogent, Canadian VIGOUR Centre, Green Lane Coordinating Centre, and South Australian Health and Medical Research Institute.

\section{REFERENCES}

1. UK Prospective Diabetes Study (UKPDS) Group. Intensive blood-glucose control with sulphonylureas or insulin compared with conventional treatment and risk of complications in patients with type 2 diabetes (UKPDS 33). Lancet 1998;352:837-53. [Erratum, Lancet 1999;354:602.]

2. Holman RR, Paul SK, Bethel MA, Matthews DR, Neil HAW. 10-Year followup of intensive glucose control in type 2 diabetes. N Engl J Med 2008;359:1577-89. 3. American Diabetes Association. Stan- dards of medical care in diabetes - 2015. Diabetes Care 2015;38:Suppl:S1-S93. 4. Nissen SE, Wolski K. Effect of rosiglitazone on the risk of myocardial infarction and death from cardiovascular causes. N Engl J Med 2007;356:2457-71. [Erratum, N Engl J Med 2007;357:100.]

5. Holman RR, Sourij H, Califf RM. Cardiovascular outcome trials of glucoselowering drugs or strategies in type 2 diabetes. Lancet 2014;383:2008-17. [Erratum, Lancet 2014;383:2212.]
6. Department of Health and Human Services, Food and Drug Administration, Center for Drug Evaluation and Research. Guidance for industry: diabetes mellitus - evaluating cardiovascular risk in new antidiabetic therapies to treat type 2 diabetes. December 2008 (http://www.fda.gov/ downloads/Drugs/GuidanceCompliance RegulatoryInformation/Guidances/ ucm071627.pdf).

7. European Medicine Agency, Committee for Medicinal Products for Human 
Use. Guideline on clinical investigation of medicinal products in the treatment of diabetes mellitus. January 2010 (http://www .ema.europa.eu/docs/en_GB/document_ library/Scientific_guideline/2010/02/ WC500073570.pdf).

8. Drucker DJ. The role of gut hormones in glucose homeostasis. J Clin Invest 2007; 117:24-32.

9. Scirica BM, Bhatt DL, Braunwald E, et al. Saxagliptin and cardiovascular outcomes in patients with type 2 diabetes mellitus. N Engl J Med 2013;369:1317-26. 10. White WB, Cannon CP, Heller SR, et al. Alogliptin after acute coronary syndrome in patients with type 2 diabetes. $\mathrm{N}$ Engl J Med 2013;369:1327-35.

11. Udell JA, Cavender MA, Bhatt DL, Chatterjee S, Farkouh ME, Scirica BM. Glucose-lowering drugs or strategies and cardiovascular outcomes in patients with or at risk for type 2 diabetes: a meta-analysis of randomised controlled trials. Lancet Diabetes Endocrinol 2015;3:356-66.

12. Clifton P. Do dipeptidyl peptidase IV (DPP-IV) inhibitors cause heart failure? Clin Ther 2014;36:2072-9.

13. Green JB, Bethel MA, Paul SK, et al. Rationale, design, and organization of a randomized, controlled Trial Evaluating Cardiovascular Outcomes with Sitagliptin (TECOS) in patients with type 2 diabetes and established cardiovascular disease. Am Heart J 2013;166:983.e7-989.e7. 14. Robins JM, Finkelstein DM. Correcting for noncompliance and dependent censoring in an AIDS Clinical Trial with inverse probability of censoring weighted (IPCW) log-rank tests. Biometrics 2000 56:779-88.
15. Egan AG, Blind E, Dunder K, et al. Pancreatic safety of incretin-based drugs - FDA and EMA assessment. N Engl J Med 2014;370:794-7. [Erratum, N Engl J Med 2014;370:2253.]

16. Zannad F, Cannon CP, Cushman WC, et al. Heart failure and mortality outcomes in patients with type 2 diabetes taking alogliptin versus placebo in EXAMINE: a multicentre, randomised, double-blind trial. Lancet 2015;385:2067-76.

17. Bethel MA, Green JB, Milton J, et al. Regional, age and sex differences in baseline characteristics of patients enrolled in the Trial Evaluating Cardiovascular Outcomes with Sitagliptin (TECOS). Diabetes Obes Metab 2015;17:395-402.

Copyright (c) 2015 Massachusetts Medical Society.

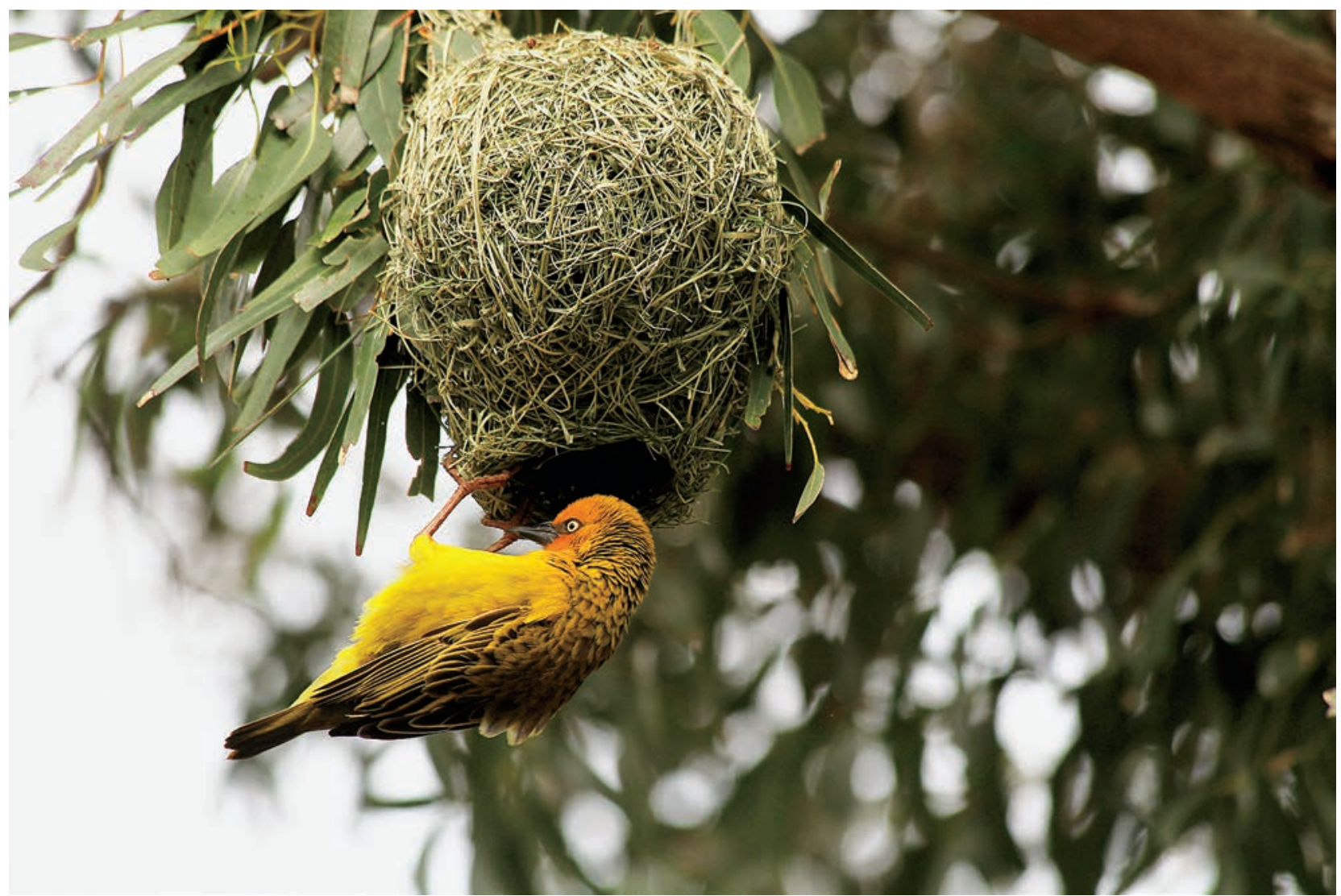

